\title{
Asociación de la variante GHRd3 del receptor de la hormona de crecimiento con autoinmunidad en la diabetes tipo 1
}

\author{
D iego García $D^{1 a^{*}}$, Amaya 0 yarzún $A^{1 b^{*}}$, \\ Bárbara Angel $\mathrm{B}^{\mathrm{1b}}$, Elena Carrasco $\mathrm{P}^{2 \mathrm{c}}$, Francisco Pérez $\mathrm{B}^{1 \mathrm{~d}}$. \\ Association of GHRd3 variant of \\ growth hormone receptor gene with \\ autoimmunity in type 1 diabetes
}

Background: Growth Hormone Receptor (GRH) is expressed in the liver, pancreas, stomach and small intestine. A high expression of GHR mRNA in the mucosal gut suggests a possible role of this receptor on digestive and immune functions. Aim: To investigate the putative effects of the GHRd3 variants on the cytokine profile and distribution of auto-antibodies in children with type 1 diabetes (T1D). Material and Methods: Unrelated unaffected controls $(\mathrm{n}=192)$ and incident cases of children with T1D $(\mathrm{n}=127)$ were analyzed for GHRd3 polymorphism, cytokine profile and a panel of auto-antibodies. Results: The allele frequency for $\mathrm{d} 3$ was $24.8 \%$ in type 1 diabetics and $34.1 \%$ in controls ( $p=N S$ ). Among type 1 diabetic children, the carriers of the GHRd3 polymorphism had significantly higher levels of interleukin-1ß than homozygous for the wild type genotype (5.7 and 17.7, $\mathrm{pg} / \mathrm{ml}$ respectively, $\mathrm{p}$ $<0.015$ ). Carriers of $d 3$ variant had a higher frequency of positive anti-insulin antibodies (antiIAA) than children without this variant (39.6 and $17.7 \%$ respectively, $p<0.01$ ). Conclusions: The observed frequency of the GHR d3/d3 genotype was comparable to other reports. A relationshiop between $\mathrm{d} 3$ variant and anti-IAA antibodies and interleukin-1ß was observed (Rev Méd Chile 2009; 137: 609-16).

(Key words: Diabetes mellitus, Type 1: Cytokines; Human growth hormone)

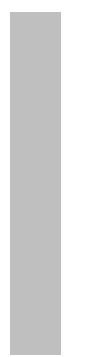

\author{
Recibido el 21 de julio, 2008. Aceptado el 23 de enero, 2009. \\ Trabajo financiado con los proyectos FONDECYT 1030680 y 1060790 \\ *La contribución de ambos autores fue similar. \\ ${ }^{1}$ Laboratorio de Epidemiología Genética, Instituto de Nutrición y Tecnología de los Ali- \\ mentos (INTA), Universidad de Chile, Santiago de Chile. ${ }^{2}$ Unidad de Diabetes, Hospital \\ San Juan de Dios, Facultad de Medicina de la Universidad de Chile, Santiago de Chile. \\ ${ }^{a}$ Candidato a Doctor en Ciencias Biológicas \\ ${ }^{\mathrm{b}}$ Candidata a Doctor en Nutrición \\ 'Nutricionista, MSc \\ dDoctor en Ciencias Biológicas
}

\footnotetext{
Correspondencia a: Dr. Francisco Pérez-Bravo. INTA- Universidad de Chile. Teléfono: 56-2-9781454, Fax: 56-2-2214030. Casilla: 138-11, Santiago-Chile.

E-mail: fperez@inta.cl
} 
L a diabetes tipo 1 (DM1) es una enfermedad autoinmune multifactorial caracterizada por la destrucción progresiva y selectiva de las células $\beta$ pancreáticas, por intermedio de factores ambientales (virus o agentes dietarios) y factores genéticos que contribuyen a la susceptibilidad al desarrollo de la enfermedad ${ }^{1}$. Dadas las características de la célula $ß$ como blanco inmunológico para la reacción autoinmune que se desarrolla en la DM1, existe una diversidad de estudios de asociación epidemiológica con otros factores tales como: el peso de nacimiento, el aumento de crecimiento postnatal, el orden de nacimiento, etc $^{2}$. Adicionalmente, el aumento de crecimiento temprano ha sido asociado a mayor sensibilidad insulínica. La DM1 mostraría, por lo tanto, un patrón de desarrollo con características inmunológicas de enfermedad autoinmune con descripción de antecedentes epidemiológicos ligados a patrones de crecimiento. En este sentido, es bien conocido el efecto de la secreción insulínica como promotora de la autoinmunidad a nivel del islote pancreático ${ }^{3}$. Desde la perspectiva del crecimiento, tanto la hormona de crecimiento $(\mathrm{GH})$, como su receptor (GHR) han sido implicados en la modulación del crecimiento y en el control metabólico de los pacientes con DM1 contribuyendo, además, a las complicaciones microvasculares de estos pacientes ${ }^{2-4}$

En humanos, el receptor de la hormona de crecimiento (GHR) existe en diversas isoformas y se expresa constitutivamente en el hígado, páncreas, estómago e intestino delgado. La isoforma GHRd3, un transcripto sin el exón 3, se expresó ampliamente y como única forma en los adenocarcinomas gástricos y colorrectales. La amplia expresión del ARN mensajero de este receptor a nivel gástrico y de mucosa intestinal ha sugerido un posible rol regulador de estas hormonas tanto sobre el sistema inmune como digestivo, incluyendo metabolismo, crecimiento o diferenciación ${ }^{5}$.

Dentro de las isoformas presentes en el GHR, se ha descrito una gran deleción en el exón 3 denominada GHRd3. La presencia de esta deleción genera dos variantes genéticas que se definen por la retención (GHRf1) o exclusión (GHRd3) del exón 3, secuencia que codifica para una región corta en el dominio extracelular del receptor. La significancia funcional de esta deleción es desconocida y publicaciones recientes sugieren que esta deleción podría ser relevante en aplicaciones en fármaco genómica de la $\mathrm{GH}^{6-8}$. Dado el posible papel de la $\mathrm{GH}$ en la patogénesis de la DM1, hemos investigado la frecuencia genotípica de esta deleción en casos incidentes de DM1 y hemos analizado su posible papel en los patrones de autoinmunidad y citoquinas asociadas a daño ß pancreático.

\section{PACIENTES Y MÉTODO}

El estudio fue realizado en Santiago, Chile, con pacientes diabéticos tipo 1 diagnosticados a través de métodos estandarizados (WHO DiaMond Project) durante el período comprendido entre enero 2004 y marzo 2006. Se incluyeron 127 casos incidentes diagnosticados durante este peńodo (edad promedio 8,5 $\pm 4,2$ años, 68 varones y 59 mujeres) y fueron comparados con 192 niños controles, sin diabetes, no relacionados (edad promedio 9,8 \pm 3,2 años, 105 varones y 87 mujeres). Todas las muestras de sangre pertenecientes a los pacientes diabéticos fueron obtenidas dentro de un corto período de tiempo cercano al debut (0 a 2 semanas). Todos los niños fueron analizados por talla al nacer y peso al nacer de acuerdo a los datos recolectados desde los archivos médicos. Todos los pacientes, controles y sus padres firmaron un consentimiento informado para participar en esta investigación. Este estudio fue aprobado por el Comité de Ética local (INTAUniversidad de Chile).

Análisis de anticuerpos. Se realizó un screening serológico para los autoanticuerpos y citoquinas séricas en 127 muestras de casos con DM1 (100\%) y en 150 muestras séricas de controles (78\%). Las mediciones de autoanticuerpos: antiácido glutámico descarboxilasa (65KD) (anti-GAD ${ }_{65}$ ), antitirosina fosfatasa (anti-IA2) y antiinsulina (IAA) fueron realizados mediante radioinmunoensayos comerciales (125I-RIA kit, DRG diagnostica, Mountainside, NJ, USA). Para los autoanticuerpos IAA se consideró una muestra "positiva" cuando el porcentaje de unión, entre el autoanticuerpo y el trazador ${ }^{125}$ I del kit fue mayor que el promedio de los controles +3 desviaciones estándar (en este caso, mayor que 5,8\%). Para antiGAD 65 y antiIA2, se designó como positivo cuando las muestras tuvieron concentraciones sobre $0,9 \mathrm{U} / \mathrm{mL}$ y $0,75 \mathrm{U} / \mathrm{mL}$, respectivamen- 
te. El panel de citoquinas séricas: IL-1ß, IL-2, IL-4, TGFß1 e INFy fueron evaluados a través del método de ELISA (R\&D system, USA).

Análisis de polimorfismo genético. De cada paciente y control se obtuvo una muestra de sangre periférica de $1 \mathrm{ml}$. El suero sobrenadante y los residuos sólidos fueron separados por centrifugación. Se utilizaron $500 \mu \mathrm{l}$ de sangre para obtener ADN genómico usando un protocolo estándar (Winkler, Santiago, Chile). Las condiciones del PCR para los tres polimorfismos fueron $95^{\circ} \mathrm{C}$ por 4 min, 30 ciclos con las siguientes características: $95^{\circ} \mathrm{C}$ por $1 \mathrm{~min}, 60^{\circ} \mathrm{C}$ por $30 \mathrm{~s} \mathrm{y} 72^{\circ} \mathrm{C}$ por $1 \mathrm{~min}, \mathrm{y}$ con una etapa de extensión final a $72^{\circ} \mathrm{C}$ por 5 min. Para determinar el genotipo del exón GHR en el locus 3 (es decir GHRfl/GHRfl, GHRfl/ GHRd3, o GHRd3/GHRd3) de cada paciente o control, realizamos un PCR con múltiple amplificación en las muestras de ADN genómico de acuerdo a lo descrito ${ }^{6}$. Brevemente, este ensayo, se fundamenta en el uso de tres partidores (dos partidores flanqueantes al exón 3 y un tercero localizado dentro de ese exón), esto permite discriminar entre los alelos GHRfl y GHRd3 ya que son amplificados como dos productos de diferente tamaño (935 pb y $532 \mathrm{pb}$, respectivamente). Al término de cada PCR verificamos la correcta amplificación en un gel de agarosa a 1,5\%. Los geles fueron visualizados mediante bromuro de etidio bajo la luz ultravioleta.
Estadística. Las comparaciones de frecuencia alélicas y genotípicas entre casos y controles fueron analizadas mediante las pruebas de Chi-cuadrado y prueba exacta de Fisher utilizando el programa SHESIS (URL: http://202.120.7.14/analysis/myAnalysis.php). Se evaluó la concordancia de las frecuencias genotípicas con respecto al equilibrio de Hardy-Weinberg mediante la prueba exacta de $\chi^{2}$. Las comparaciones de las variables continuas del estudio se realizaron mediante prueba $t$ de Student, la comparación del perfil de anticuerpos se realizó a través de análisis de proporciones. Un valor de $p$ menor de 0,05 fue considerado como estadísticamente significativo.

\section{RESULTADOS}

La Tabla 1 resume las características antropométricas de ambos grupos de acuerdo a la característica genotípica GHRd3 (portador de la variante f1/f1 y portadores de la variante d3). Se observaron diferencias estadísticamente significativas al comparar entre portadores y no portadores de la variante $\mathrm{d} 3$ en los niños con DM1 con respecto al peso al nacer $(3,02 \pm 0,025 \mathrm{~kg}$ en $\mathrm{f1} / \mathrm{f1}$ versus 2,81 $\pm 0,275 \mathrm{~kg}$ en portadores $\mathrm{d} 3, \mathrm{p}<0,01)$ y la talla al debut $(1,35 \pm 0,27 \mathrm{~cm}$ en $\mathrm{f} 1 / \mathrm{fl}$ versus $1,25 \pm 0,035$ $\mathrm{cm}$ en portadores d3, p <0,012). En el grupo control no se observaron diferencias significativas entre estas dos categorías genotípicas.

Tabla 1. C aracterísticas antropométricas de ambos grupos de estudio, de acuerdo al genotipo G H Rd3

\begin{tabular}{|lccc|}
\hline Grupo D M $\mathbf{1}$ & Genotipo f1/f1 & Portadores d3 & p-value \\
\hline Edad (años) & $8,94 \pm 3,5$ & $8,11 \pm 0,5$ & NS \\
Peso al nacer $(\mathrm{kg})$ & $3,02 \pm 0,025$ & $2,81 \pm 0,275$ & 0,01 \\
Talla al nacer $(\mathrm{cm})$ & $0,50 \pm 0,01$ & $0,49 \pm 0,015$ & NS \\
Peso al debut $(\mathrm{kg})$ & $35,2 \pm 15,5$ & $31,4 \pm 3,5$ & NS \\
Talla al debut $(\mathrm{cm})$ & $1,35 \pm 0,27$ & $1,25 \pm 0,035$ & 0,012 \\
\hline Grupo control & Genotipo f1/f1 & Portadores d3 & p-value \\
\hline Edad (años) & $10,3 \pm 2,5$ & $9,3 \pm 3,8$ & NS \\
Peso al nacer $(\mathrm{kg})$ & $2,95 \pm 0,015$ & $2,84 \pm 0,075$ & NS \\
Talla al nacer $(\mathrm{cm})$ & $0,49 \pm 0,02$ & $0,48 \pm 0,01$ & NS \\
Peso actual $(\mathrm{kg})$ & $37,8 \pm 8,3$ & $37,9 \pm 8,2$ & NS \\
Talla actual $(\mathrm{cm})$ & $1,39 \pm 7,3$ & $1,40 \pm 6,1$ & NS \\
\hline
\end{tabular}


La frecuencia alélica para el alelo d3 fue de $24,8 \%$ para los DM1 y de 34,1\% para los controles ( $p=N S$ ). Las observaciones del genotipo GHRd3 se resumen en la Tabla 2. La población analizada en este estudio mostró una frecuencia para el alelo d3 comparable con los valores publicados en la literatura, a excepción de lo publicado en población de Israel y Polonia (Tabla 2). Además, al igual que en otras poblaciones ${ }^{6,8,9}$ no se observó equilibrio de Hardy-Weinberg para la muestra analizada.

En relación al perfil de autoanticuerpos, todos los niños controles fueron negativos para el panel de autoanticuerpos antiGAD ${ }_{65}$, antiIA-2 y antiIAA. Entre los niños con DM1, no se detectaron diferencias en cuanto a antiGAD ${ }_{65}$ y antiIA-2 por género o por grupos de edad al diagnóstico (0-4 años; 5-9 años o 10-14 años). Sin embargo, el perfil de autoanticuerpos antiIAA fue significativamente mayor cuando se comparó por grupos de edad: en los grupos de edad de 0-4 años y de 5-9 años el porcentaje positivo de antiIAA fue $39 \%$ y $30 \%$, respectivamente, comparado con $10 \%$ observado en el grupo 10-14 años ( $p<0,02)$.

Al comparar la distribución del perfil de autoanticuerpos en relación con la variante GHRd3, se observó una diferencia significativa para los niveles positivos de antiIAA entre los portadores de la variante d3 (39,6\%) y los no portadores de d3 $(17,7 \%)$, ( $p<0,012)$. No se observaron diferencias para la distribución de anticuerpos antiGAD65 y antiIA-2 a través de los genotipos f1/f1 o portadores de GHRd3 (Tabla 3).

Finalmente, entre los niños con DM1, se observaron dos características asociadas al perfil de citoquinas. Por un lado, el polimorfismo d3 fue asociado con niveles significativamente altos de IL-1ß (valor promedio 19,5 versus $8,9 \mathrm{pg} / \mathrm{ml}, \mathrm{p}<0,015) \mathrm{y}$ aquellos niños portadores del genotipo f1/f1 mostraron elevadas concentraciones de IL-2 (Figura 1).

Tabla 2. Frecuencias de genotipo GH Rd3 en niños chilenos diabéticos tipo 1 y niños controles comparados con datos previamente publicados

\begin{tabular}{|c|c|c|c|c|c|c|c|}
\hline \multirow[t]{2}{*}{ Población } & \multicolumn{2}{|c|}{$\mathbf{f 1 / f 1}$} & \multicolumn{2}{|c|}{ f1/d3 } & \multicolumn{2}{|c|}{ d3/d3 } & \multirow{2}{*}{$\begin{array}{r}d 3 \\
(\%)\end{array}$} \\
\hline & n. & Frec. & n. & Frec. & n. & Frec. & \\
\hline $\begin{array}{l}\text { Niños con DM1 } \\
(\mathrm{n}=127)\end{array}$ & 79 & 0,622 & 33 & 0,259 & 15 & 0,118 & 24,8 \\
\hline $\begin{array}{l}\text { Niños control } \\
(n=192)\end{array}$ & 97 & 0,505 & 59 & 0,307 & 36 & 0,188 & 34,1 \\
\hline $\begin{array}{l}\text { Niños SGA Europa } \\
(\mathrm{n}=76)\end{array}$ & 36 & 0,473 & 24 & 0,316 & 16 & 0,211 & 36,8 \\
\hline $\begin{array}{l}\text { Niños ISS Europa }{ }^{8} \\
(\mathrm{n}=76)\end{array}$ & 50 & 0,521 & 38 & 0,396 & 8 & 0,083 & 35,5 \\
\hline $\begin{array}{l}\text { Niños ISS Alemania } 9 \\
(\mathrm{n}=77)\end{array}$ & 32 & 0,416 & 33 & 0,428 & 12 & 0,156 & 37,0 \\
\hline $\begin{array}{l}\text { Niños ISS Israel }{ }^{22} \\
(\mathrm{n}=30)\end{array}$ & 16 & 0,533 & 9 & 0,300 & 5 & 0,167 & 31,6 \\
\hline $\begin{array}{l}\text { Población Israel } 22 \\
(\mathrm{n}=82)\end{array}$ & 39 & 0,476 & 9 & 0,110 & 34 & 0,414 & 46,9 \\
\hline $\begin{array}{l}\text { Población Francia } 6 \\
(\mathrm{n}=150)\end{array}$ & 88 & 0,587 & 49 & 0,327 & 13 & 0,086 & 25,0 \\
\hline $\begin{array}{l}\text { Población Polonia }{ }^{21} \\
(\mathrm{n}=526)\end{array}$ & 272 & 0,517 & 221 & 0,420 & 33 & 0,063 & 54,5 \\
\hline $\begin{array}{l}\text { Niños LBW Chile }{ }^{10} \\
(\mathrm{n}=38)\end{array}$ & 20 & 0,526 & 14 & 0,368 & 4 & 0,106 & 26,3 \\
\hline
\end{tabular}

SGA = Pequeños para la edad gestacional; ISS = Corta estatura idiopática; LBW = Pequeño peso nacimiento. 
Tabla 3. Portadores GH Rd3 y perfil de auto-anticuerpos en niños con D M 1 (valores positivos de anti-IAA, anti-IA-2 y anti-G $A_{65}$ )

\begin{tabular}{|lrrrrrr|}
\hline GHRd3 & \multicolumn{2}{c}{ IAA+ } & \multicolumn{2}{c}{ IA-2+ } & \multicolumn{2}{c|}{ GAD $_{65}^{+}$} \\
& $\mathbf{n}$ & $\%$ & $\mathbf{n}$ & $\%$ & $\mathbf{n}$ & $\%$ \\
\hline f1/f1 (n=79) & 14 & 17,7 & 55 & 69,6 & 57 & 72,1 \\
Portadores d3 $(\mathrm{n}=48)$ & 19 & $39,6^{*}$ & 32 & 66,6 & 39 & 81,3 \\
\hline
\end{tabular}

$*_{p}<0,012$.

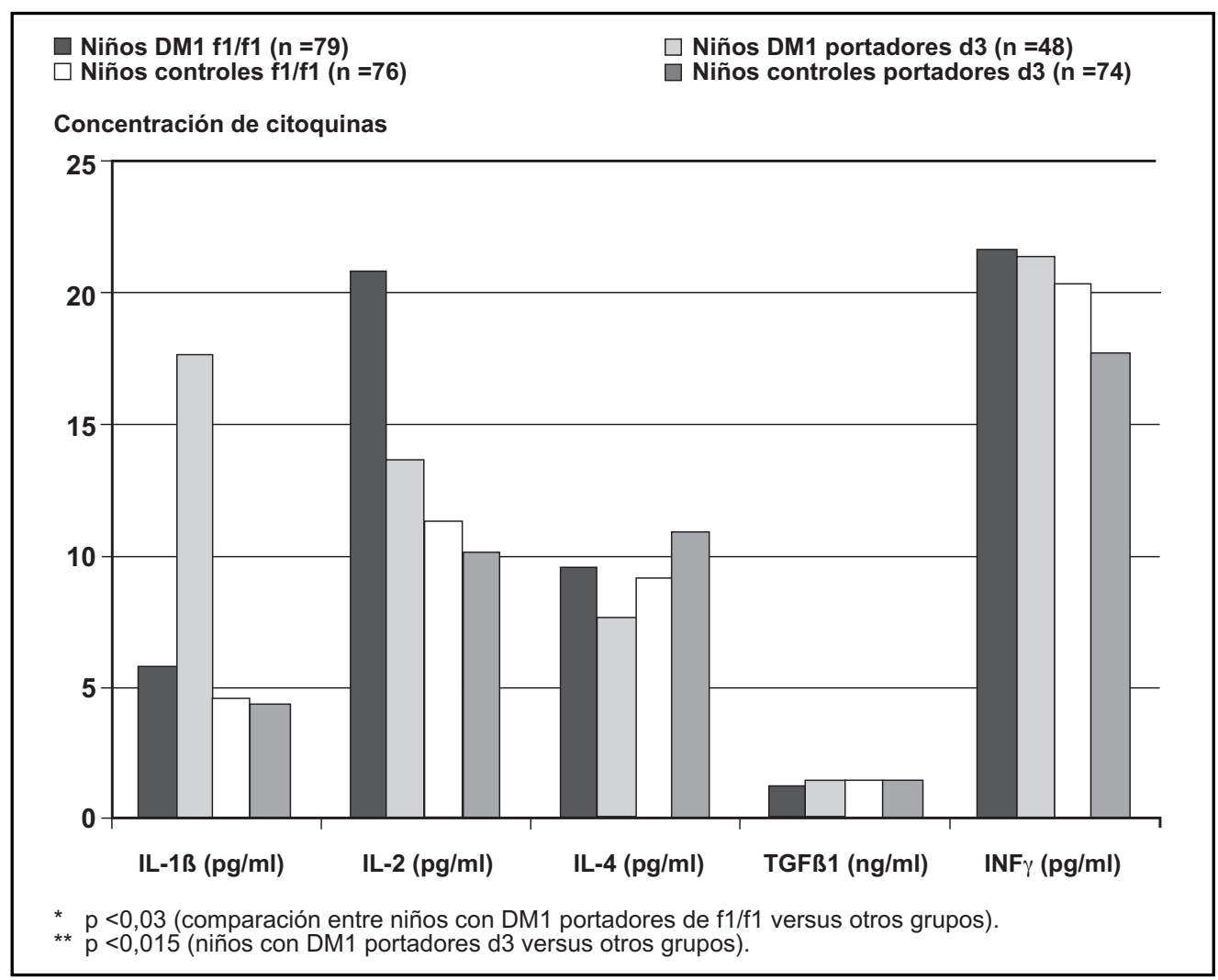

Figura 1. Portadores GHRd3 y nivel de citoquinas en niños diabéticos tipo 1 y niños sanos (mediana).

\section{DISCUSIÓN}

La expresión generalizada del ARN mensajero del receptor $\mathrm{GH}$ en líneas celulares gástricas y de mucosa, en particular en epitelios, ha sugerido un posible papel regulador de estas hormonas en funciones digestivas e inmunes ${ }^{5}$. La relación entre el sistema neuroendocrino y el sistema inmune se ha propuesto desde hace mucho tiempo y se conoce que las hormonas pituitarias, incluyendo la $\mathrm{GH}$, afectan la respuesta inmune. La GH estimula la síntesis de ADN en linfocitos $\mathrm{T}$ humanos y aumenta la actividad citotóxica de las células natural killer (NK), además es capaz de estimular la proliferación normal y leucémica de los linfocitos T humanos ${ }^{3}$.

Los resultados obtenidos en este estudio, muestran que el polimorfismo GHRd3 es bastante 
frecuente y común en nuestra población $(24,8 \%$ en DM1 y 34,1\% en controles, $p=N S$ ), cifra comparable al único estudio publicado hasta la fecha en población de América Latina ${ }^{10}$. En forma similar a otras publicaciones, nuestros datos por lo menos en población con DM1, muestran una posible asociación de esta variante genotípica (portadores d3) con características ligadas al bajo peso al nacer y baja talla.

La muestra de pacientes y controles analizada no se encontró en equilibrio de Hardy-Weinberg, situación que podría tener implicancias respecto a la representatividad de la muestra tomada. No obstante, al menos otros tres estudios publicados en la literatura ${ }^{6,8,9}$ muestran un fenómeno similar. $\mathrm{Al}$ respecto, existen varias posibles explicaciones para la existencia de asociaciones alélicas y las desviaciones a esta ley. Una posibilidad es que la población esté estratificada en las muestras, esta situación claramente apuntaría a un sesgo de selección ya sea en los pacientes con DM1 o en controles. Esto corresponde a una preocupación frecuente en los estudios basados en poblaciones, por lo que los tamaños de muestra resultan críticos en estudios de epidemiología genética. Sin embargo, esta misma población (de casos y controles) ha sido analizada para al menos cinco genes asociados a DM1 como HLA clase II, receptor de vitamina D (VDR), CTLA-4 y PD-1, donde se ha observado equilibrio y sólo ha mostrado desequilibrio en 2 variantes, una para VDR y otra para PD-111-14. Debemos señalar que este polimorfismo es bastante sencillo de determinar y al ser un sistema de exclusión, la posibilidad de error de asignación de alelos es muy baja ya que la resolución no genera solapamientos de bandas. La desviación del equilibrio de HardyWeinberg también podría indicar que los marcadores genéticos utilizados se encuentran próximos a algún gen relacionado con una enfermedad, situación que debe ser evaluada con futuros estudios.

Se ha demostrado que la GH tiene un potente papel en el sistema inmune. Sin embargo, los mecanismos involucrados sobre sus efectos en este sistema aún no han sido clarificados ${ }^{8}$. En los pacientes con DM1, encontramos una asociación importante entre los anticuerpos antiIAA, la concentración de IL-1ß y la condición de ser portador de la variante GHRd3, en comparación con los no portadores (f1/f1). En este sentido, se podría especular un posible papel de este polimorfismo genético en los niveles de citoquinas a través de una vía de señalización apoptótica en el entorno de la célula beta.

Varios estudios han sugerido que la GH regula el sistema inmunológico humano a través de la inhibición de la apoptosis tanto en linfocitos $\mathrm{T}$, como en células B activadas. Desde la perspectiva de los marcadores de apoptosis, las caspasas inflamatorias han sido propuestas como el posible vínculo entre el sistema inmune innato y las enfermedades de origen autoinmune. Se ha documentado que tanto la activación de caspasa-1, como de caspasa-5 tienen relación con los altos niveles de citoquinas como la IL-1 ß e IL-18. Además, el aumento de la actividad de caspasa 3 se asocia a un desbalance entre IL-1 ß e INFy ${ }^{15-17 .}$ Existe el antecedente del único estudio con un modelo de autoinmunidad (casos de cáncer asociados a retinopatía, CAR), donde los autoanticuerpos (anti-recoverina) que son citotóxicos para las células de la retina inducen la muerte apoptótica por la activación una vía apoptótica caspasa-3 dependiente $^{18}$. En DM1 la vía de caspasa 3 está siendo profusamente estudiada y al parecer esta ruta es capaz de modular la respuesta apoptótica del linfocito $\mathrm{T}$ autorreactivo que infiltra al islote ${ }^{19,20}$.

En Latinoamérica sólo existe un estudio sobre este polimorfismo genético ${ }^{10} \mathrm{y}$ los restantes once estudios publicados son de origen europeo, por lo que las comparaciones de frecuencia han resultado difíciles a la hora de analizar los tamaños muestrales involucrados en cada estudio (Tabla 2) y la carencia de datos en otras patologías que no fueran aquellas relacionadas a crecimiento 6,8,21,22.

Nuestros resultados exploran por primera vez una posible relación entre el efecto de las variantes de GHRd3 y la autoinmunidad en la DM1. A pesar de que la frecuencia de la variante d3 no fue diferente entre pacientes y controles, la observación en el grupo de pacientes con DM1, de asociaciones con peso al nacer, talla al debut y perfil de anticuerpos y citoquinas podrían permitir especular una posible asociación de este polimorfismo con desregulación del sistema inmunológico a nivel de linfocitos $\mathrm{T}$, evento que debiera ser analizado en el futuro a través de estudios in vitro con células mononucleares por ejemplo ${ }^{23}$. 
Este análisis describe por primera vez la frecuencia de la variante $\mathrm{d} 3$ en pacientes con DM1. El principal hallazgo de nuestro estudio ha sido sugerir que el genotipo GHRd3 podría tener efectos sobre los perfiles de citoquinas o autoanticuerpos circulantes. Si bien el mecanismo es bastante especulativo, la expresión de este receptor en líneas celulares gastrointestinales lo podría ligar a funciones inmunológicas a nivel de maduración de la mucosa ${ }^{5}$. La mucosa intestinal y en especial la microbiota que allí se aloja ha sido intensamente estudiada en el último tiempo y en la actualidad acapara la atención de estudios pioneros en el área ${ }^{24,25}$.

La DM1 es una enfermedad pancreática endógena, donde las células $ß$ se han perdido debido a un complejo proceso de destrucción autoinmune. Varios factores de crecimiento se han aplicado en islotes aislados in vitro, sin mostrar un efecto relevante en un aumento significativo de la población de células $ß$. La alteración completa del receptor de hormona de crecimiento causa disminución del islote pancreático por lo que se supone es esencial en la cascada de señalización que regula finalmente el tamaño celular26-28.

Publicaciones recientes continúan mostrando asociaciones diversas de esta variante en distintas patologías, como cáncer de mama, donde d3 sería protector y sensibilidad insulínica con resultados

\section{REFERENCIAS}

1. KaWasaki E, Abiru N, Eguchi K. Prevention of type 1 diabetes: from the view point of $\AA$ cell damage. Diab Res and Clin Pract 2004; 66: 27-32.

2. THRAILKIL KM. Insulin-like growth factor-I in diabetes mellitus: its physiology, metabolic effects, and potential clinical utility. Diab Technol Ther 2000; 2: 6980.

3. BRUINING GJ. Association between infant growth before onset of juvenile type 1 diabetes and autoantibodies to IA-2. Lancet 2000; 356: 655-6.

4. HaLdin M, Brismar K, Tuvemo T, Gustafsson J. Insulin sensitivity and lipolysis in adolescent girls with poorly controlled type 1 diabetes: effect of anticholinergic treatment. Clin Endocrinol 2002; 57: 735-43.

5. Nagano M, Chastre E, Choquet A, Bara J, Gespach C, KELY PA. Expression of prolactin and growth hormone receptor genes and their isoforms in the gastrointestinal tract. Am J Physiol 1995; 268: G431G442. contradictorios $^{29,30}$. La evidencia indica que tanto GH como su receptor (GHR) están implicados directamente en una serie de procesos metabólicos e inmunológicos y que su acción la ejercerían fundamentalmente a través de una serie de cascadas de señalización que tienen un impacto directo en proliferación celular, diferenciación, migración, prevención de la apoptosis, reorganización del citoesqueleto y regulación metabólica ${ }^{31}$. Sería muy útil realizar estudios funcionales para dilucidar la real influencia de este polimorfismo en el mecanismo del crecimiento pancreático de las células $ß$ (neogénesis o replicación de las células $ß$ existentes del islote), que permita aclarar si esta variante tiene un papel en la vías de señalización intracelular responsables de la proliferación de las células $ß$ mediada por glucosa (mitogénesis) o si esta variable realmente incide en un patrón de autoinmunidad en las primeras etapas de la DM1 donde el mecanismo responsable del desbalance inmunológico sigue siendo una interrogante.

\section{Agradecimientos}

Los autores agradecen la colaboración de todos los niños y sus familias participantes en este estudio. A los Hospitales San Juan de Dios, San Borja-Arriarán y a la Fundación de Diabetes Juvenil de Chile.

6. Pantel J, Machinis K, Sobrier ML, Duquesnoy P, Goossens M, Amselem S. Species-specific alternative splice mimicry at the growth hormone receptor locus revealed by the lineage of retroelements during primate evolution. J Biol Chem 2000; 275: 18664-9.

7. Pantel J, Grulich-Henn J, Bettendorf M, Strasburger CJ, HeinRich U, Amselem S. Heterozygous nonsense mutation in exon 3 of the growth hormone receptor (GHR) in severe GH insensitivity (Laron Syndrome) and the issue of the origin and function of the GHRd3 isoform. J Clin Endocrinol Metab 2003; 88: 1705-10.

8. Dos Santos C, Essioux L, Teinturier C, Tauber M, GofFin V, Bougneres P. A common polymorphism of the growth hormone receptor is associated with increased responsiveness to growth hormone. Nat Genet 2004; 36: 720-4.

9. Schreiner F, Stutte S, Bartman P, Gohlke B, Woelfie J. Association of the growth hormone receptor d3variant and catch-up growth of preterm infants with 
birth weight of less than 1500 grams. J Clin Endocrinol Metab 2007; 92: 4489-93.

10. MericQ V, Román R, Iñiguez G, Angel B, Salazar T, AviLA A ET AL. Relationship between nocturnal growth hormone concentrations, serum IGF-I/IGFBP-3 levels, insulin sensitivity and $\mathrm{GH}$ receptor allelic variant in small for gestational age children. Horm Res 2007; 68: 132-8.

11. Pérez-Bravo F, Santos Jl, Carrasco E, Calvillan M, Albala C, Puig-Domingo M, De Leiva A. Assessment of the interaction between autoantibodies (ICA and GAD65) with HLA-DQB1 alleles in type 1 diabetes through a case-parental study conducted in Santiago, Chile. Autoimmunity 2001; 33: 285-91.

12. López T, García D, Angel B, Carrasco E, Codner E, Ugarte F, PÉrez-Bravo F. Asociación del polimorfismo Fok I del receptor de vitamina D (VDR) con niveles plasmáticos TGF-ß1 e INF- $\gamma$ en la diabetes mellitus tipo 1. Med Clin (Barcelona) 2008; 130: 81-4.

13. García D, Angel B, Carrasco E, Albala C, Santos JL, PÉREZ-BRAVo F. VDR polymorphisms influence the immune response in type 1 diabetic children from Santiago, Chile. Diab Res Clin Pract 2007; 77: 13440.

14. Momim S, Flores S, Angel B, Codner E, Carrasco E, PÉrez-Bravo F. Interaction between programmed death 1 (PD-1) and cytotoxic T lymphocyte antigen 4 (CTLA-4) gene polymorphisms in type 1 diabetes. Diab Res Clin Pract 2008 (in press).

15. Mitsunaka $\mathrm{H}$, Dobashi $\mathrm{H}$, Sato M, Tanaka T, Kitanaka A ET AL. Growth hormone prevents Fas-induced apoptosis in lymphocytes through modulation of Bcl-2 and caspase-3. Neuroimmunomodulation 2001; 9: 256-62.

16. Bohm SA, Wolf M, Kreymann G, Holscher AH. Proinflammatory cytokines IL-1 beta and TNF-alpha reduce growth hormone receptor mRNA concentration in cultivated rat hepatocytes after stimulation with growth hormone. Langenbecks Arch Chir 1998; 115: 185-8.

17. Augstein P, Bahr J, Wachlin G, Heinke P, Berg S, SAlzsieder E, Harrison LC. Cytokines activate caspase3 in insulinoma cells of diabetes-prone NOD mice directly and via upregulation of Fas. J Autoimmun 2004; 23: 301-9.

18. Adamus G. Auto-antibody-induced apoptosis as a possible mechanism of autoimmune retinopathy. Autoimmun Rev 2003; 2: 63-8.

19. Vendrame F, Santangelo C, Misasi R, Dionisi S, Gizzi C, REAlacci M ET AL. Defective lymphocyte caspase-3 expression in type 1 diabetes mellitus. Eur J Endocrinol 2005; 152: 119-25.
20. Vendrame F, Segni M, Grassetti D, Tellone V, Augelo G, TRISChITTA V ET AL. Impaired caspase-3 expression by peripheral $\mathrm{T}$ cells in chronic autoimmune thyroiditis and in autoimmune polyendocrine syndrome-2. J Clin Endocrinol Metab 2006; 7: 5064-8.

21. Wagner K, Hemminki K, Grzybowska E, Bermejo JL, Butkiewicz D, Pamula J et al. Polymorphisms in the growth hormone receptor: a case-control study in breast cancer. Int J Cancer 2006; 118: 2903-6.

22. Hujeirat Y, Hess O, Shalev S, Tenenbaum-Rakover Y. Growth hormone receptor sequence changes do not play a role in determining height in children with idiopathic short stature. Hormone Res 2006; 65: 21016.

23. Chiarewi F, Giannini C, Mohn A. Growth, growth factors and diabetes. Eur J Endocrinol 2004; 151: 109-17.

24. Vaarala O, AtKinson MA, Neu J. The perfect storm for type 1 diabetes: The complez interplay between intestinal microbiota, gut permeability and mucosal immunity. Diabetes 2008; 57: 2555-62.

25. Wen L, Ley RE, Volchkov Py, Stranges PB, Avanesyan L, StONEBRAKER AC ET AL. Innate immunity and intestinal microbiota in the development of type 1 diabetes. Nature 2008; 455: 1109-13.

26. Carter-Su C, Smit LS. Signalling via JAK tyrosine kinase: growth hormone receptor as a model system. Recent Progress Hor Research 1998; 53: 61-82.

27. RHodes CJ. IGF-I and GH post-receptor signalling mechanism for pancreatic $ß$-cell replication. J Mol Endocrinol 2000; 24: 303-11.

28. Lu JL, Coschigano KT, Robertson K, Lipsett M, Guo Y, Kо PCHICK JJ ET AL. Disruption of growth hormone receptor gene cause diminished pancreatic islet size and increased insulin-sensitivity in mice. Am J Physiol Endocrinol Metab 2004; 287: E405-E413.

29. Toyoshima MTK, Castroneves LA, Costalonga EF, Mendonca BB, Arnhold JP, Jorge AAL. Exon 3-deleted genotype of growth hormone receptor (GHRd3) positively influences IGF-1 increase at generation test in children with idiopathic short stature. Clin Endocrinol 2007; 67: 500-4

30. Schmid C, Krayenbuehl PA, Bernays RL, Zwimpfer C, MaLy FE, WiesLi P. Growth hormone (GH) receptor isoform in acromegaly: lower concentrations of $\mathrm{GH}$ but not insulin-like growth factor-1 in patients with a genomic deletion of exon 3 in the $\mathrm{GH}$ receptor gene. Clin Chem 2007; 53: 1484-8.

31. Lanning NJ, Carter-Su C. Recent advances in growth hormone signalling. Rev Endocr Metab Disord 2006; 7: 225-35. 